proper double points for nodes. If we project the projected surface upon an $S_{3}$, we have an $F^{\prime 4 n-4}$ having a double curve of order $8 n^{2}-23 n+17$ with $16 n-28$ pinch points. If the center of projection is in $\pi$, the double curve degenerates into a $(4 n-6)$ fold line and $3 n-4$ double lines. Since the class of $K^{4 n-6}$ is $10 n-20$, there are on the $(4 n-6)$-fold line $10 n-20$ pinch points. The remaining $6 n-8$ pinch points are on the $3 n-4$ double lines, 2 on each.

The University of California

\title{
SPINORS AND TENSORS
}

BY G. Y. RAINICH

It is well known that there are two kinds of quantities connected with the representations of a group of rotations-the tensors and the spinors. ${ }^{*}$ Since the advent of the relativity theory we had been led to believe, in the words of O. Veblen, $\dagger$ "that any physical phenomena could be described by means of tensors." But then came the Dirac equations of the electron which give an example of a situation described in terms of spinors. Does it mean that we have to change the belief expressed above? It does not follow. All that has happened is that we have a phenomenon not described in terms of tensors; that does not mean that it cannot be-so described. That it might be possible to describe every situation given in spinors also in tensors is suggested by the fact that there exist algebraic relations between spinors and tensors; it may be possible to eliminate the spinors from a sufficient number of these algebraic relations and the given spinor differential equations, and obtain in this way an equivalent description in tensors. The discussion of the general case should not be very difficult, but it seemed that a simple special case should be worked out first, and that is why I suggested to Gordon Fuller the problem which he discusses in his article. $\ddagger$ The problem there is treated without

* Compare, for example, R. Brauer and H. Weyl, American Journal of Mathematics, vol. 40 (1935), p. 425.

$\dagger$ Proceedings of the National Academy of Sciences, vol. 24 (1934), p. 282.

$\ddagger$ This issue of this Bulletin, vol. 42 (1936), p. 107. 
reference to a physical situation, and, so to say, as an isolated case, and possibly may appear artificial. The following remarks may help to establish the proper perspective.

A. The four auxiliary quantities $\alpha, \beta, \gamma, \delta$ which appear in Fuller's article may be combined into two complex numbers

$$
\phi=\alpha+i \beta, \quad \psi=\delta+i \gamma,
$$

and then the components of the vector $F$ and the scalar $\rho$ are expressed as follows:

$$
\begin{array}{ll}
u=\phi^{*} \psi+\psi^{*} \phi, & v=\phi^{*} \psi-\psi^{*} \phi, \\
w=\phi^{*} \phi-\psi^{*} \psi, & \rho=\phi^{*} \phi+\psi^{*} \psi,
\end{array}
$$

where $\phi^{*}$ denotes the complex conjugate of $\phi$. The differential equations (6) of Fuller's paper may be written as

$$
\frac{\partial \phi}{\partial x}+i \frac{\partial \phi}{\partial y}-\frac{\partial \psi}{\partial z}=\frac{1}{2} \psi, \quad \frac{\partial \psi}{\partial x}-i \frac{\partial \psi}{\partial y}+\frac{\partial \phi}{\partial z}=\frac{1}{2} \phi
$$

the generalized equations ( 8 ) would be obtained from these by replacing the operators $\partial / \partial x, \partial / \partial y, \partial / \partial z$ by $\partial / \partial x-i l, \partial / \partial y-i m$, and $\partial / \partial z-i n$, respectively.

B. Once the equations are written in this form the analogy with the Dirac equations is seen immediately. In fact, the equations (2) may be obtained as a special case from the Dirac equations if we set in these latter ones (we take them in the form given by Weyl) $\dagger$

$$
\psi_{1}=\psi_{3}=\phi, \quad \psi_{2}=-\psi_{4}=\psi,
$$

assume that these quantities are independent of $z$, and change the notations of coordinates as follows:

$$
\frac{2 m c}{h} x \text { to } y, \frac{2 m c}{h} y \text { to } x \text {, and } \frac{2 m c^{2} i}{h} t \text { to } z \text {. }
$$

C. In particular, we notice that gauge transformation applies to this case exactly in the same way as in the general case; this corresponds to the appearance of the angles $\Omega$ and then $\theta$ in Fuller's work. 171 .

$\dagger$ Gruppentheorie und Quantenmechanik, 1st ed., 1928, formulas (45'), p. 
D. The general problem of expressing spinor equations in tensor form, mentioned in the beginning of this article, may be formulated for the special case we are considering as follows: eliminate $\phi$ and $\psi$ from the system (1), (2). Fuller solves this problem and arrives at the following result. The system (1), (2) is equivalent to the vector equation

$$
\rho^{3} \operatorname{curl} \frac{1}{\rho} \operatorname{curl} F=V,
$$

where $\rho^{2}=F^{2}$ and the components of $V$ are given by the determinants

$$
\left|\begin{array}{lll}
u & v & w \\
u_{y} & v_{y} & w_{y} \\
u_{z} & v_{z} & w_{z}
\end{array}\right|, \quad\left|\begin{array}{ccc}
u & v & w \\
u_{z} & v_{z} & w_{z} \\
u_{x} & v_{x} & w_{x}
\end{array}\right|, \quad\left|\begin{array}{lll}
u & v & w \\
u_{x} & v_{x} & w_{x} \\
u_{y} & v_{y} & w_{y}
\end{array}\right| .
$$

E. A more detailed discussion will be postponed until the general Dirac system has been translated into tensor form, but it should be stated now that it is not proposed to replace the use of spinor equations in Physics by tensor equations. The spinor equations (2) have the great advantage of being linear, whereas the corresponding vector equations (3) are badly nonlinear. In fact, had the equations (3) been given first, the transition to (2) would have been a very important step in their study. We might call this transition linearization, using this term in a sense different from that given it by Dirac.

UNIVERSITY OF MichigAN 